\title{
Asymmetry Coefficients as Indicators of Chaos: Hyperchaotic Qi System
}

\author{
P. WĄż $\dot{Z}^{a, *}$ AND D. BIELIŃSKA-WĄż $\dot{Z}^{b}$ \\ ${ }^{a}$ Centrum Astronomii, Uniwersytet Mikołaja Kopernika, Gagarina 11, 87-100 Toruń, Poland \\ ${ }^{b}$ Instytut Fizyki, Uniwersytet Mikołaja Kopernika, Grudziądzka 5, 87-100 Toruń, Poland
}

(Received October 31, 2012)

\begin{abstract}
Statistical properties of the hyperchaotic Qi system are studied. The theory, recently formulated and applied for the damped driven pendulum, is used in this investigation. Asymmetry coefficients, related to the statistical moments of distributions composed from the time-series, are shown to behave in a different way for periodic, chaotic and hyperchaotic solutions and are proposed as indicators of chaos and hyperchaos.
\end{abstract}

DOI: 10.12693/APhysPolA.123.647

PACS: 02.70.Rr, 95.10.Fh, 05.45.-a, 05.45.Gg, 05.45.Tp

\section{Introduction}

Studies on hyperchaos, which may appear in at least four-dimensional autonomous systems, become popular in science and engineering [1-8]. Hyperchaotic systems are characterized by more than one positive Lyapunov exponent. Generally, hyperchaos is more disordered than chaos and that gives advantages of hyperchaotic systems, in particular in secure communication $[9,10]$. The frequency spectrum of hyperchaos is broader than that of the ordinary chaos and, consequently, can cover a broader spectrum of the message.

The first hyperchaotic system was reported by Rössler [11]. Since then, also other hyperchaotic systems were found [12-21].

In the present work, we consider the statistical indicators of chaos and hyperchaos for the recently introduced hyperchaotic system [22]. We call this system from the name of its author "the Qi system". The authors carry out frequency spectral analysis, which demonstrates that the system has an extremely broad high-magnitude frequency band width, very desirable for engineering applications as e.g. secure communication.

We continue our statistical studies which we have already applied for a creation of indicators of chaos [23]. We have already applied analogous statistical methodology in other areas of science: in astrophysics [24, 25], in the theory of molecular similarity [26-29], and in bioinformatics [30].

The studies on indicators of chaos were the subject of many investigations [31-39]. Our alternative indicator, already applied to the damped driven pendulum [23], is simple for coding and can be directly applied to the observational data (to the time-series). Generally, hyperchaos is more disordered than the ordinary chaos and our indicators (the coefficients describing the asymmetry of the

*corresponding author; e-mail: snake@umk.pl time-series) are expected to reveal also this property (see subsequent sections).

\section{Method}

Let us consider the time-series $X(t)$ that describes a time evolution of a quantity characterizing the system. Let us define $K$ sections

$$
X^{k}(t) \equiv\left\{X(t), t \in\left\langle T_{s}, T_{f}^{k}\right\rangle\right\}, \quad k=1,2, \ldots K
$$

with $T_{f}^{1}<T_{f}^{2}<\ldots T_{f}^{K}$. All $k$ sections have the same origin $T_{s}$. Let us define the length of the $k$-th section

$$
\Delta T^{k}=T_{f}^{k}-T_{s} .
$$

We treat $X^{k}(t)$ as statistical distributions. Their lengths increase

$$
\Delta T^{1}<\Delta T^{2}<\ldots \Delta T^{K} .
$$

Usually, in practical applications, $X(t)$ is known from experimental measurements. Then, its values are given in a discrete set of points $\left\{\tau_{i}\right\}_{i=1}^{L}$. Usually, the experimental points are equidistant

$$
\tau_{i+1}-\tau_{i}=\delta=\text { const. }
$$

In the $k$-th section corresponding to the time interval $\left\langle T_{s}, T_{f}^{k}\right\rangle, k=1,2, \ldots, K$, we have the following measurement points:

$$
\tau_{i} \equiv t_{i}^{k}=T_{s}+\mathrm{i} \delta, i=1, \ldots, N_{k}
$$

with $t_{1}^{k}=T_{s}$ and $t_{N_{k}}^{k}=T_{f}^{k}$. $N_{k}$ is the number of points in the $k$-th section and $N_{1}<N_{2}<\ldots N_{K}$.

The asymmetry coefficients of the $k$-th distribution $X^{k}(t)$ are defined as

$$
\begin{aligned}
& M_{q}^{\prime \prime}\left(k, N_{k}\right)=\mathcal{N}\left(k, N_{k}\right) \sum_{i=1}^{N_{k}}\left(X_{i}^{k}+c\right) \\
& \quad \times\left[\frac{t_{i}^{k}-M_{1}\left(k, N_{k}\right)}{\sqrt{M_{2}\left(k, N_{k}\right)-M_{1}\left(k, N_{k}\right)^{2}}}\right]^{q},
\end{aligned}
$$

where

$$
\mathcal{N}\left(k, N_{k}\right)=\left[\sum_{i=1}^{N_{k}}\left(X_{i}^{k}+c\right)\right]^{-1},
$$




$$
M_{n}\left(k, N_{k}\right)=\mathcal{N}\left(k, N_{k}\right) \sum_{i=1}^{N_{k}}\left(X_{i}^{k}+c\right)\left(t_{i}^{k}\right)^{n},
$$

$n=0,1,2,3, \ldots, q=3$, and $c$ is a numerical constant introduced to avoid singularity in Eq. (6). As it is shown in the next section, the qualitative character of the results does not depend on $c$ in a relatively broad range of values of this parameter.

The asymmetry of the distribution is described by $M_{3}^{\prime \prime}$. Higher order moments $(q>4)$ do not have a direct geometrical interpretation. Very often, the odd moments of order higher than 3 i.e. $M_{q}^{\prime \prime}, q=5,7,9, \ldots$ are correlated with $M_{3}^{\prime \prime}[29]$. Though $M_{5}^{\prime \prime}$ and $M_{7}^{\prime \prime}$ contain similar information to $M_{3}^{\prime \prime}$, they can be also considered in studies on the symmetry of the distributions.

If the distribution is symmetric, then $M_{3}^{\prime \prime}$, called skewness, is zero. Generally, evenly distributed points on both sides of the mean may give a zero value of the skewness also for distributions which are not exactly symmetric. A skewness value is negative if the left tail of the distribution is longer, and it is positive if the right one is longer. In the present work we consider the distributions with many maxima. As it is shown in the next section, the time-series for periodic cases are more symmetric than for the chaotic and hyperchaotic ones and are characterized by $M_{3}^{\prime \prime}$ close to zero.

The theory can be easily extended to the case when the density of the measurement points $\delta$ is not constant, i.e. Eq. (4) is not fulfilled. In such case, Eqs. (6)-(8) are exactly the same and the summations are performed over the given points. Moreover, continuous $X(t)$ can also be considered by replacing the summations by the appropriate integrals [23].

As shown in the next section, the dependence of the asymmetry coefficients on the lengths of $X^{k}(t)$ (i.e. also on $N_{k}$ which is proportional to $\Delta T^{k}$ ) is different for periodic, chaotic, and hyperchaotic cases. For $\Delta T^{1}$ smaller than periods the asymmetry coefficients can be quite large also in periodic cases. The symmetry is recognized when the lengths of the sections is sufficiently large. Therefore, with lengths of the sections increasing, the asymmetry coefficients converge rapidly to zero. In chaotic and hyperchaotic cases some sections may also exhibit signs of symmetry. Then the coefficients may also be close to zero. However, generally, their concentration around zero is smaller than in periodic cases. The mean value of $M_{3}^{\prime \prime}$ :

$$
D=\frac{1}{K} \sum_{k=1}^{K}\left|M_{3}^{\prime \prime}\left(k, N_{k}\right)\right|
$$

may be taken as a measure of the concentration around zero. As it is shown in the next section, $D$ is the smallest for periodic cases and the largest for the hyperchaotic ones.

\section{Results and discussion}

In the present work, we consider recently introduced $4 \mathrm{D}$ hyperchaotic system which is described by the fol- lowing system of equations [22]:

$$
\left.\begin{array}{l}
\dot{x}_{1}=a\left(x_{2}-x_{1}\right)+x_{2} x_{3}, \\
\dot{x}_{2}=g\left(x_{1}+x_{2}\right)-x_{1} x_{3}, \\
\dot{x}_{3}=-c x_{3}-e x_{4}+x_{1} x_{2}, \\
\dot{x}_{4}=-d x_{4}+f x_{3}+x_{1} x_{2} .
\end{array}\right\}
$$

The time-series $X(t) \equiv x_{1}(t)$ has been derived from these equations. The calculations are performed for the following parameters: $a=50, c=13, d=8, e=33$, $f=30$. Six different values of parameters $g$ are taken. For $g=0.1$ and $g=0.45$ the solutions are periodic. For $g=2.15$ and $g=13$ the solutions are chaotic, and for $g=23$ and $g=26$ the solutions are hyperchaotic [22].

The differential equations have been solved numerically. The main part of the numerical integration code constitutes the procedure RA15 [40]. The calculations have been performed for the time sequence $\left\{\tau_{i}\right\}_{i=1}^{2000}$ with a step $\delta=0.1$, and $\tau_{1}=0.1$.

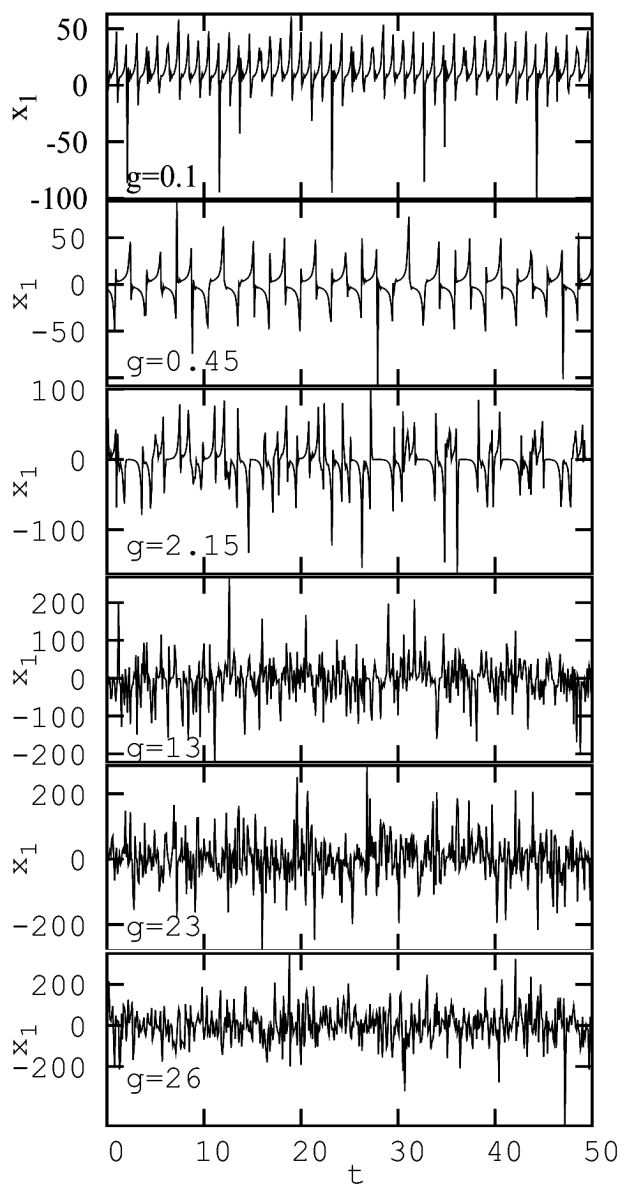

Fig. 1. Time-series $x_{1}(t)$ for the Qi system for different values of $g$.

The distributions are composed of $x_{1}(t)$ which is shown in Fig. 1. The origin of each distribution corresponds to the initial time $T_{s}=\tau_{1}$ (fixed for all distributions). The number of points in the shortest distribution $N_{1}=100$ and $N_{k}-N_{k-1}=100$ where $k=1,2, \ldots 20$. That corre- 
sponds to the number of points in the longest distribution $N_{\mathrm{K}}=2000$.

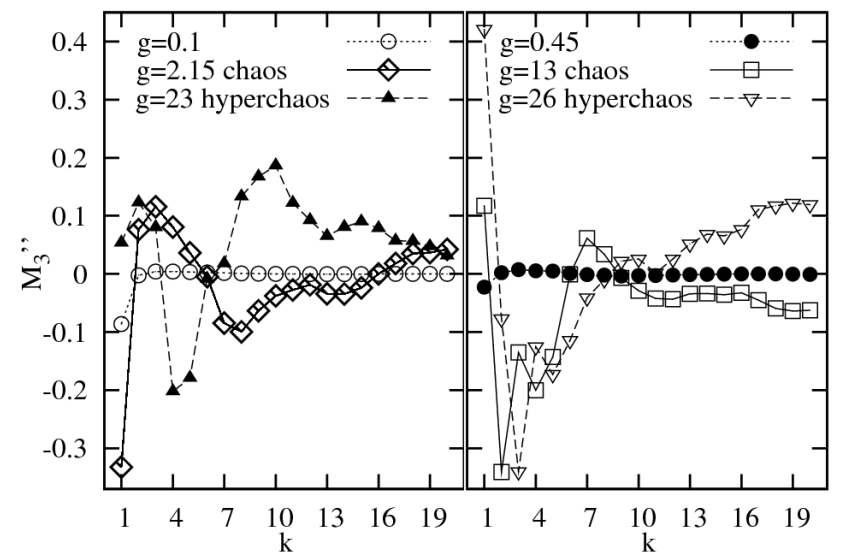

Fig. 2. $\quad M_{3}^{\prime \prime}$ for different values of $g$.

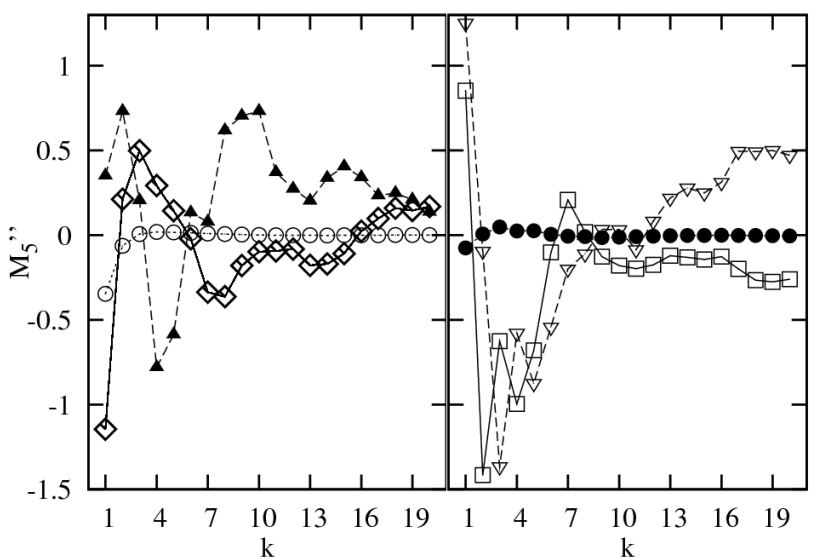

Fig. 3. $\quad M_{5}^{\prime \prime}$ for different values of $g$. The symbols are the same as in Fig. 2.

Figures $2-4$ show $M_{3}^{\prime \prime}, M_{5}^{\prime \prime}, M_{7}^{\prime \prime}$ for $k=1,2, \ldots 20$. The parameter $c=30$. The pattern in each figure is similar to each other, and that suggests correlations between $M_{3}^{\prime \prime}$, $M_{5}^{\prime \prime}$, and $M_{7}^{\prime \prime}$. In all the figures for a short distribution, i.e. for $N_{k}=100(k=1)$ the symmetry for the periodic cases is not recognized, and the coefficients are far from zero. However, on increasing $k$ (for longer distributions), zero is reached quickly. For chaotic and hyperchaotic cases, the variations of the coefficients are much larger.

Figure 5 shows $D$, i.e. the average module of $M_{3}^{\prime \prime} . D$ is approximately zero for periodic solutions (circles). It is larger for chaos (squares), and the largest for hyperchaos (triangles). $D$ is shown for several values of $c$ and the qualitative results are the same.

Summarizing, the presented method due to its simplicity is convenient to identify symptoms of chaos and hyperchaos. The asymmetry coefficients for the hyperchaotic Qi system are close to zero. They can also distinguish between chaos and hyperchaos.

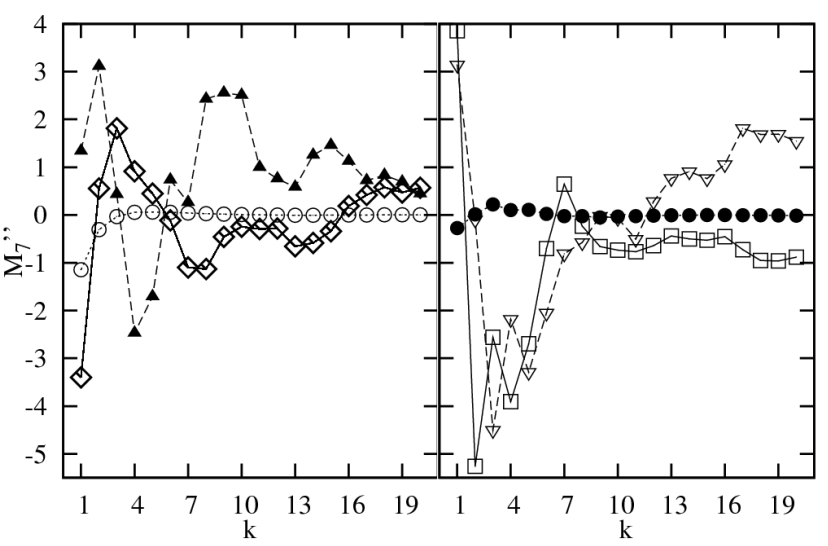

Fig. 4. $\quad M_{7}^{\prime \prime}$ for different values of $g$. The symbols are the same as in Fig. 2.

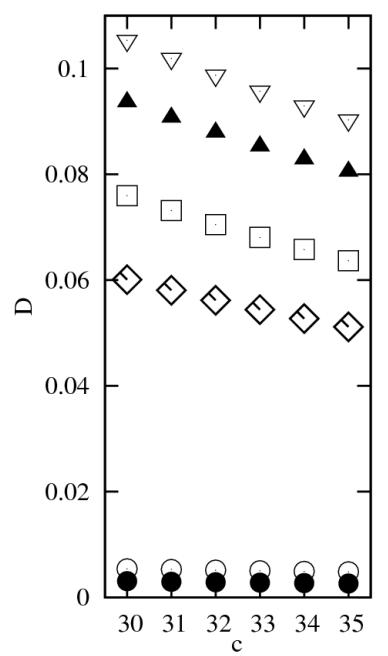

Fig. 5. $D$ for different $c$. The symbols are the same as in Fig. 2.

\section{References}

[1] G. Qi, M.A. van Wyk, B.J. van Wyk, G. Chen, Chaos Solitons Fractals 40, 2544 (2009).

[2] C. Yang, C.H. Tao, P. Wang, Phys. Lett. A 374, 729 (2010).

[3] S. Dadras, H.R. Momeni, Phys. Lett. A 374, 1368 (2010).

[4] S. Zheng, X. Han, Q. Bi, Commun. Nonlinear Sci Numer. Simulat. 16, 1998 (2011).

[5] K.P. Harikrishnan, R. Misra, G. Ambika, Commun. Nonlinear Sci. Numer. Simulat. 17, 263 (2012).

[6] E.E. Mahmoud, J. Franklin Inst. 349, 1247 (2012).

[7] M.J. Correia, P.C. Rech, Appl. Math. Comput. 218, 6711 (2012).

[8] C. Li, Commun. Nonlinear Sci. Numer. Simulat. 17, 405 (2012) 
[9] G. Grassi, S. Mascolo, IEEE Trans. Circuits Syst. I 46, 1135 (1999).

[10] V.S. Udaltsov, J.P. Goedgebuer, L. Larger, J.B. Cuenot, P. Levy, W.T. Rhodes, Opt. Spectrosc. 95, 114 (2003).

[11] O.E. Rössler, Phys. Lett. A 71, 155 (1979).

[12] T. Kapitaniak, L.O. Chua, Int. J. Bifurc. Chaos 4, 477 (1994).

[13] K. Thamilmaran, M. Lakshmanan, A. Venkatesan, Int. J. Bifurc. Chaos 14, 221 (2004).

[14] Y. Li, G. Chen, W.K.S. Tang, IEEE Trans. Circuits Syst. II 52, 204 (2005).

[15] R. Barboza, Int. J. Bifurc. Chaos 17, 4285 (2007).

[16] X. Wang, M. Wang, Physica A 387, 3751 (2008).

[17] Q. Yang, K. Zhang, G. Chen, Nonlinear Anal. RWA 10, 1601 (2009).

[18] Q. Yang, Y. Liu, J. Math. Anal. Appl. 360, 293 (2009).

[19] Y. Liu, Q. Yang, G. Pang, J. Comput. Appl. Math 234, 101 (2010).

[20] Y. Li, W.K.S. Tang, G. Chen, Int. J. Circuit Theory Appl. 33, 235 (2005).

[21] S. Pang, Y. Liu, J. Comput. Appl. Math. 235, 2775 (2011).

[22] G. Qi, M.A. van Wyk, B.J. van Wyk, G. Chen, Phys. Lett. A 372, 124 (2008).

[23] P. Wąż, D. Bielińska-Wąż, Acta Phys. Pol. A 116 , 987 (2009).

[24] P. Wąż, D. Bielińska-Wąż, A. Pleskacz, A. Strobel, Acta Phys. Pol. B 39, 1993 (2008).

[25] P. Wąż, D. Bielińska-Wąż, A. Strobel, A. Pleskacz, Acta Astron. 60, 283 (2010).
[26] D. Bielińska-Wąż, P. Wąż, S.C. Basak, Eur. Phys. J. B 50,333 (2006).

[27] D. Bielińska-Wąż, P. Wąż, S.C. Basak, J. Math. Chem. 42, 1003 (2007).

[28] D. Bielińska-Wąż, P. Wąż, J. Math. Chem. 43, 1287 (2008).

[29] D. Bielińska-Wąż, W. Nowak, Ł. Pepłowski, P. Wąż, S.C. Basak, R. Natarajan, J. Math. Chem. 43, 1560 (2008).

[30] D. Bielińska-Wąż, W. Nowak, P. Waż, A. Nandy, T. Clark, Chem. Phys. Lett. 443, 408 (2007).

[31] G. Contopoulos, N. Voglis, Astron. Astrophys. 317, 73 (1997).

[32] C. Froeschlé, E. Lega, Astron. Astrophys. 334, 355 (1998).

[33] M. Gutzwiller, Chaos in Classical and Quantum Mechanics, Springer Verlag, New York 1990.

[34] G. Benettin, L. Galgani, A. Giorgilli, J.M. Strelcyn, Meccanica 15, 9 (1980); 15, 21 (1980).

[35] A. Wolf, J.B. Swift, H.L. Swinney, J.A. Vastano, Physica D 16, 285 (1985).

[36] C. Piccardi, Chaos 14, 1026 (2004); 16, 043115 (2006), and references therein

[37] Z. Sándor, B. Érdi, A. Széll, B. Funk, Celest. Mech Dyn. Astr. 90, 127 (2004).

[38] Ch. Skokos, T.C. Bountis, Ch. Antonopoulos, Physica D 231, 30 (2007).

[39] Ch. Skokos, T.C. Bountis, Ch. Antonopoulos, Eur. Phys. J. ST 165, 5 (2008).

[40] E. Everhart, Celest. Mech. 10, 35 (1974). 